\title{
Effect of the Operation Mode and Distance on the Electromagnetic Radiation Emitted by Mobile Phone Devices in Greece: A Pilot Study
}

Dionysios Koulougliotis ${ }^{1}$, Dimitrios Nikolopoulos ${ }^{2 *}$, Nikolaos Gorgolis ${ }^{2}$, Lefteris Karidas ${ }^{2}$, Ermioni Petraki ${ }^{2}$ and Panayiotis H Yannakopoulos ${ }^{2}$

${ }^{1}$ Department of Environmental Technology, School of Technological Applications, Technological Educational Institute (TEI) of Ionian Islands, Greece

${ }^{2}$ Department of Electronic Computer Systems Engineering, School of Technological Applications, Piraeus University of Applied Sciences (TEl of Piraeus), Greece

\begin{abstract}
The electromagnetic radiation (EMR) emitted by mobile phone devices $(n=52)$ has been monitored as a function of the operation mode and the distance from the device. Measurements (electric field, $\mathrm{V} / \mathrm{m}$ ) were administered in the metropolitan area of Athens, Greece and involved a total of 52 mobile phone devices operating in the $1.5 \mathrm{GHZ}-2.1$ $\mathrm{GHz}$ frequency range. Five distinct operation modes of the device ("long term inactivity", "ringing/call effort", "usage", "right after the end of the call", "short term inactivity") and a total of two distances from the device $(0 \mathrm{~m}$ and $1 \mathrm{~m})$ were employed. Depending on the intensity of the individual peaks observed in the experimental spectra at $0 \mathrm{~m}$, the mobile devices were shown to cluster into three subgroups as follows: Subgroup $1\left(n_{1}=24\right.$, peak intensities $\left.>1 \mathrm{~V} / \mathrm{m}\right)$, Subgroup $2\left(n_{2}=9\right.$, peak intensities in the range of $\left.0.1 \mathrm{~V} / \mathrm{m}-1 \mathrm{~V} / \mathrm{m}\right)$, Subgroup $3\left(n_{3}=19\right.$, peak intensities $\left.<0.1 \mathrm{~V} / \mathrm{m}\right)$. Statistical analysis via repeated-measures analysis of variance (RM-ANOVA) provided evidence for a statistically significant effect of the operation mode on the emitted EMR by the mobile device. The effect was more prominent at the shortest distance $(0 \mathrm{~m})$ and for the devices of one specific subgroup (Subgroup 1). The transition between operation modes which shows the largest effect (increase) in the emitted EMR is the one from "long term inactivity" to "Ringing/Call effort" irrespective of device subgroup. Examination of the effect of the distance on the emitted EMR resulted to the following findings: At the longer distance employed $(1 \mathrm{~m})$, the devices belonging to Subgroups 2 and 3 continue to exhibit mean EMR intensity similar to the one at $0 \mathrm{~m}$ under all operation modes. On the other hand, upon distance increase, the emitted EMR intensity of the devices belonging to Subgroup 1 displays a statistically significant decrease in all operation modes except the one of "long term inactivity".
\end{abstract}

Keywords: Mobile phones; Electromagnetic radiation

\section{Introduction}

It is well established that the recent progress in communications has raised significant concerns about the potential adverse health effects due to the exposure to the associated electromagnetic radiation (EMR) [19]. The human exposure depends on the electromagnetic field strength, the distance from the EMR emitting device and the proximity to the directional antennas [10-30]. The uncontrolled rise of devices and antennas of modern life increased significantly the EMR sources in the human environment and hence, the associated exposure.

Recognising the potential human risks, the Council of the European Union (CEU) recommended limitations for the EMR for frequencies between $0 \mathrm{~Hz}$ and $300 \mathrm{GHz}[22,23]$ (Table 1). The suggested reference levels for these frequencies focus on limiting the strength of the EMR field in the environment and the electrical contact or induced currents in the human body. In 2013, the European Parliament and the CEU published the Directive 2013/35/EU [23] regarding the exposure of EU citizens to EMR. Despite that the Recommendations [22,23] do not bind the EU member states [24], they direct the member states to set strict occupational exposure limits and to investigate EMR around power lines, mobile phone base stations and any other sources accessible to the general public in order to assure the compliance with the Recommendations. On the other hand, the Scientific Committee on Emerging and Newly Identified Health Risks (SCENIHR) [6-8] utilises different frequency bands for the protection from EMR: (i) RF EMR $(100 \mathrm{kHz}<\mathrm{f} \leq 300 \mathrm{GHz})$; (ii) intermediate frequency (IF) EMR (300 $\mathrm{Hz}<\mathrm{f} \leq 100 \mathrm{kHz}$ ), (iii) ELF EMR $(0 \mathrm{~Hz}<\mathrm{f} \leq 300 \mathrm{~Hz})$; (iv) static fields (0 $\mathrm{Hz})$ and (iv) Tera frequency EMR $\left(0.3 \times 10^{12} \mathrm{~Hz}<\mathrm{f} \leq 3 \times 10^{12} \mathrm{~Hz}\right)$ due to expected increase in use of $\mathrm{THz}$ technologies in the next years.

The International Commission on Non-Ionizing Radiation Protection (ICNIRP) utilizes also different frequency range for the protection to EMR. The guidelines issued in 2010 referred to frequencies between $1 \mathrm{~Hz}$ and $100 \mathrm{kHz}$ [26], while those of 2009 referred to frequencies between $100 \mathrm{kHz}$ and $300 \mathrm{GHz}$ [27], reconfirming the validity of guidelines of 1998 [28]. The ICNIRP Recommendations for the general public around mobile phone base stations are presented in Table 2 [4,26-57]. Regarding the estimation of human exposure to RF EMR ICNIRP suggests measurement averaging over time to address cumulative effects [25-28]. For RF EMR between $3 \mathrm{kHz}$ and $100 \mathrm{kHz}$, the averaging time should be 1 second to estimate the induced and contact currents. For RF EMR fields between $100 \mathrm{kHz}$ and $15000 \mathrm{MHz}$, the averaging time should be of some minutes, so as the temperature increase in human tissue to be detectable, while for EMR between 100

\begin{tabular}{|c|c|c|}
\hline Frequency & Type & Source \\
\hline $0 \mathrm{~Hz}-300 \mathrm{kHz}$ & $\begin{array}{c}\text { Extremely low frequency (LF- } \\
\text { ELF) to Low frequency (LF) } \\
\text { electromagnetic radiation }\end{array}$ & $\begin{array}{c}\text { Electrical fields of devices, } \\
\text { conventional electrical } \\
\text { network, video }\end{array}$ \\
\hline $\begin{array}{c}3 \mathrm{kHz}-300 \\
\mathrm{MHz}\end{array}$ & Radio frequencies (RF) & $\begin{array}{c}\text { Sections of AM radio, FM } \\
\text { radio }\end{array}$ \\
\hline $\begin{array}{c}300 \mathrm{MHz}-300 \\
\mathrm{GHz}\end{array}$ & Microwave (MW) & $\begin{array}{c}\text { Mobile telephones, DECT } \\
\text { devices, radar and other } \\
\text { microwave communications }\end{array}$ \\
\hline
\end{tabular}

Table 1: Classification of EMR sources according to the Council of the European Union.

*Corresponding author: Dimitrios Nikolopoulos, Department of Electronic Computer Systems Engineering, School of Technological Applications, Piraeus University of Applied Science, Petrou Ralli and Thivon 250, GR-12244, Aigaleo, Greece, Tel: 0030-210-5381560; Fax: 0030-210-5381436; E-mail: dniko@teipir.gr

Received August 09, 2017; Accepted February 15, 2018; Published February 20, 2018

Citation: Koulougliotis D, Nikolopoulos D, Gorgolis N, Karidas L, Petraki E, et al. (2018) Effect of the Operation Mode and Distance on the Electromagnetic Radiation Emitted by Mobile Phone Devices in Greece: A Pilot Study. J Civil Environ Eng 8: 300. doi: 10.4172/2165-784X.1000300

Copyright: $\odot 2018$ Koulougliotis D, et al. This is an open-access article distributed under the terms of the Creative Commons Attribution License, which permits unrestricted use, distribution, and reproduction in any medium, provided the original author and source are credited. 
Citation: Koulougliotis D, Nikolopoulos D, Gorgolis N, Karidas L, Petraki E, et al. (2018) Effect of the Operation Mode and Distance on the Electromagnetic Radiation Emitted by Mobile Phone Devices in Greece: A Pilot Study. J Civil Environ Eng 8: 300. doi: 10.4172/2165784X.1000300

Page 2 of 9

\begin{tabular}{|c|c|c|c|}
\hline Frequency & $\begin{array}{c}\text { Electric field strength } \\
(\mathbf{V} / \mathbf{m})\end{array}$ & $\begin{array}{c}\text { Magnetic field } \\
\text { strength }(\boldsymbol{\mu T})\end{array}$ & $\begin{array}{c}\text { Power density } \\
\mathbf{W} / \mathbf{m}^{2}\end{array}$ \\
\hline $900 \mathrm{MHz}(\mathrm{GSM})$ & 41 & 0.14 & 4.5 \\
\hline $\begin{array}{c}1800 \mathrm{MHz} \\
\text { (DCS) }\end{array}$ & 58 & 0.2 & 9 \\
\hline $\begin{array}{c}2100 \mathrm{MHz} \\
\text { (UMTS) }\end{array}$ & 61 & 0.2 & 10 \\
\hline
\end{tabular}

Table 2: ICNIRP Recommendations for the general public for EMR fields of frequencies around mobile phone base stations.

\begin{tabular}{|c|c|c|}
\hline Operation Mode & Total Mean EMR value (V/m) & Std. Dev. (V/m) \\
\hline OM1 & 0.03 & 0.025 \\
\hline OM2 & 0.159 & 0.15 \\
\hline OM3 & 0.214 & 0.197 \\
\hline OM4 & 0.264 & 0.175 \\
\hline OM5 & 0.264 & 0.137 \\
\hline
\end{tabular}

Table 3: Descriptive Statistics for Subgroup $1(\mathrm{~N}=24$, Distance $=0 \mathrm{~m})$.

$\mathrm{kHz}$ and $6 \mathrm{GHz}$ [25] it recommends the use of the Specific Absorption Rate (SAR).

Taking into account the above issues and the international interest regarding the potential health effects of the EMR [1-35], this paper focused on RF EMR measurements of the mobile telephony frequency range by examining the following two specific research questions:

i) What is the dependence (if any) of the EMR intensity emitted by the mobile phone device on the operation mode of the device?

ii) What is the dependence (if any) of the EMR intensity of the mobile phone device on the distance from the device?

It should be pointed out that this is a pilot study which was conducted in a sample of 52 mobile devices and as such it aims to serve as a basis for extensive RF EMR measurements in Greece.

\section{Materials and Methods}

Measurements of electromagnetic radiation (EMR) emitted by 52 mobile phones were obtained at two specific distances from the device (namely $0 \mathrm{~m}$ and $1 \mathrm{~m}$ ), by employing the NARDA SRM-3006 instrument. The mobile devices were set to function in five different operation modes $(\mathrm{OM})$ and measurements were taken for all five OMs at each specific distance. The five operation modes were the following:

Operation mode 1- OM1: Long term inactivity

Operation mode 2- OM2: Ringing/Call effort (either incoming or outgoing call)

Operation mode 3- OM3: Usage (During the conversation)

Operation mode 4- OM4: Right after the end of the call

Operation mode 5- OM5: Short term inactivity (shortly after the end of the call)

Each measurement of the NARDA SRM-3006 instrument consists of three dynamic spectra (Electric Field $(\mathrm{V} / \mathrm{m})$ vs Frequency $(\mathrm{GHz})$ ) within a user-defined frequency range. Each spectrum is made up of 244 data points (distributed in equally spaced frequency bins) which correspond to the Maximum, Minimum and Average EMR value (Electric Field) at a predefined measuring time period. The present paper reports the results obtained from the analysis of the spectra corresponding to the maximum EMR values at the frequency range between $1.5 \mathrm{GHz}$ and $2.1 \mathrm{GHz}$ for a period of two minutes, after which, the mobile telephony companies in Greece terminate automatically the operation modes 2, 4 and 5. From each derived spectrum, the mean electric field (mean of all maxima) was calculated and was subsequently employed as the characteristic one describing the electromagnetic radiation emitted by the specific device under the specific measurement condition (OM and distance).

The statistical test Repeated Measures ANOVA (RM-ANOVA) was employed in order to examine differences between the mean EMR values (mean of all maxima) under the five different operation modes. It was applied by using the Greenhouse-Geisser correction for the violation of the assumption of sphericity and the Bonferroni correction for posthoc tests in order to get results from specific pairwise comparisons. In order to examine differences between two mean values (as in the case of the two distances) the paired samples t-test was employed together with the non-parametric Wilcoxon signed-rank test as ancillary. The assumption of normality in the data distribution was examined via the application of the Kolmogorov-Smirnov test at the 95\% significance level (i.e. $p>0.05$ in order to accept that data are normally distributed). In all comparisons made, statistically significant differences at the $95 \%$ significance level are reported. All statistical tests were made via the use of the SPSS software.

\section{Results}

\section{EMR intensity of mobile devices at $0 \mathrm{~m}$}

Initially, the distribution shape of each one of the five whole data sets of mean EMR values (one whole set for each of the five OMs) taken at a distance of $0 \mathrm{~m}$, was examined. All five whole data sets (i.e. data sets comprised of all 52 mean EMR values) were shown not to follow a normal distribution which does not allow the application of statistical tests (related to analysis of variance) for examining the existence of certain trends in the data. This result pointed out the need to examine the possibility of dividing the data from the 52 devices in subgroups so that the condition of normality is simultaneously satisfied for all five OM sub-data sets of mean EMR values (OM1-OM5) within each subgroup. A careful examination of the data obtained from all 52 devices in all five OM sub-data sets via the systematic application of the KolmogorovSmirnov test for normality, resulted to the division of the data in three different subgroups. Within each subgroup, all five OM sub-data sets satisfied the Kolmogorov-Smirnov criterion for normality. The main criterion for the setup of the subgroups has been the intensity of the peaks observed in the individual spectra of the devices. As it will be shown subsequently, in the three identified subgroups the intensities of the observed peaks (in at least one of the five OMs) fall in the following ranges: $>1 \mathrm{~V} / \mathrm{m}$ (Subgroup 1), between $0.1 \mathrm{~V} / \mathrm{m}$ and $1 \mathrm{~V} / \mathrm{m}$ (Subgroup 2) and $<0.1 \mathrm{~V} / \mathrm{m}$ (Subgroup 3).

More specifically, the three identified subgroups were the following:

Subgroup 1: It included twenty-four (24) mobile devices. The mean EMR values of the devices in this subgroup were in the range between $0.007 \mathrm{~V} / \mathrm{m}$ and $0.82 \mathrm{~V} / \mathrm{m}$ under all OMs. In this subgroup at least one of the observed mean EMR values (in some OM) was larger than $0.09 \mathrm{~V} / \mathrm{m}$. The individual spectra in at least one of the five OMs displayed large peaks (between $1 \mathrm{~V} / \mathrm{m}$ and $10 \mathrm{~V} / \mathrm{m}$ ) for all 24 devices and even higher than $10 \mathrm{~V} / \mathrm{m}$ for eight of them. In general, the mean EMR values of this subgroup showed large changes as a function of the OM employed. More specifically, maximal changes ranging by factors between 2.47 and 45.8 were observed. In fact, half of the devices showed maximal changes by factors between 2 and 10 and the other half by even larger factors (between 10 and 45.8).

The main descriptive statistics for the data of Subgroup 1 at a distance of $0 \mathrm{~m}$ from the device are given in Table 3. All mean EMR values are below the limit values of Table 2 . 
Citation: Koulougliotis D, Nikolopoulos D, Gorgolis N, Karidas L, Petraki E, et al. (2018) Effect of the Operation Mode and Distance on the Electromagnetic Radiation Emitted by Mobile Phone Devices in Greece: A Pilot Study. J Civil Environ Eng 8: 300. doi: 10.4172/2165784X.1000300

Page 3 of 9

In order to test for statistically significant differences between the total mean EMR values as a function of the operation mode, the Repeated Measures ANOVA (RM-ANOVA) test was applied. The necessity to apply RM-ANOVA emerges from the fact that we are interested in examining differences between mean values exhibited by the same mobile devices under more than two different conditions and specifically under five different OMs.

The RM-ANOVA showed the existence of a statistically significant effect of the operation mode on the EMR intensity emitted by the mobile devices belonging to Subgroup 1 at distance equal to $0 \mathrm{~m}(F(1.871$, $43.039)=31.103, p<0.001)$. The pairwise comparisons via post-hoc tests (Bonferroni correction) showed the following specific statistically significant differences:

OM1 $<$ OM2 $(p=0.002), \mathrm{OM} 3(p=0.001), \mathrm{OM} 4(p<0.001)$ and OM5 $(p<0.001)$

\section{OM2 $<$ OM3 $(p=0.02)$, OM4 $(p<0.001)$ and OM5 $(p<0.001)$}

More specifically, there is a statistically significant increase in total mean EMR intensity by ca. a factor of 5.30 on passing from the OM1 to $\mathrm{OM} 2$ and a further increase by ca. a factor of 1.35 on passing from OM2 to OM3. Upon passing from OM3 to OM4 to OM5 the EMR intensity remained at a constant high level displaying no statistically significant changes. The above pairwise comparisons may be summarised as follows:

\section{$\mathrm{OM} 1<\mathrm{OM} 2<\mathrm{OM} 3 \approx \mathrm{OM} 4 \approx \mathrm{OM} 5$}

In Figure 1, the mean EMR values obtained for the 24 mobile devices of Subgroup 1 are depicted in the form of Box-Whisker plots together with the results of the statistical analysis via RM-ANOVA. The mode of presentation is the following: for each data set (operation mode), its code name (OM1-OM5) is shown in bold accompanied in parenthesis by the code name(s) of the other(s) data set(s) with which it shows no statistically significant difference $(p>0.05)$. If a data set happens to be statistically different from all others, its code name is reported alone.

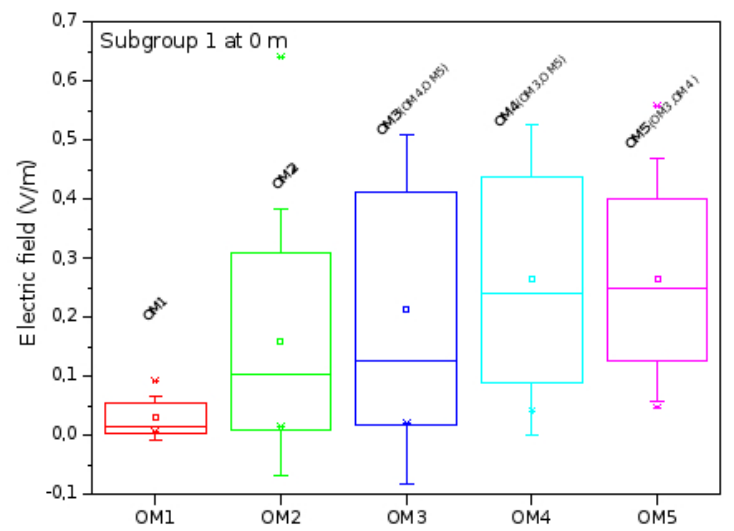

Figure 1: Box-Whisker plots of EMR intensity at $0 \mathrm{~m}$ for the twenty-four (24) mobile devices of Subgroup 1 at the five different operation modes (OM1 to OM5).

\begin{tabular}{|c|c|c|}
\hline Operation Mode & Total Mean EMR value (V/m) & Std. Dev. (V/m) \\
\hline OM1 & 0.043 & 0.018 \\
\hline OM2 & 0.055 & 0.024 \\
\hline OM3 & 0.058 & 0.024 \\
\hline OM4 & 0.054 & 0.023 \\
\hline OM5 & 0.054 & 0.022 \\
\hline
\end{tabular}

Table 4: Descriptive statistics for subgroup $2(\mathrm{~N}=9$, Distance $=0 \mathrm{~m})$.

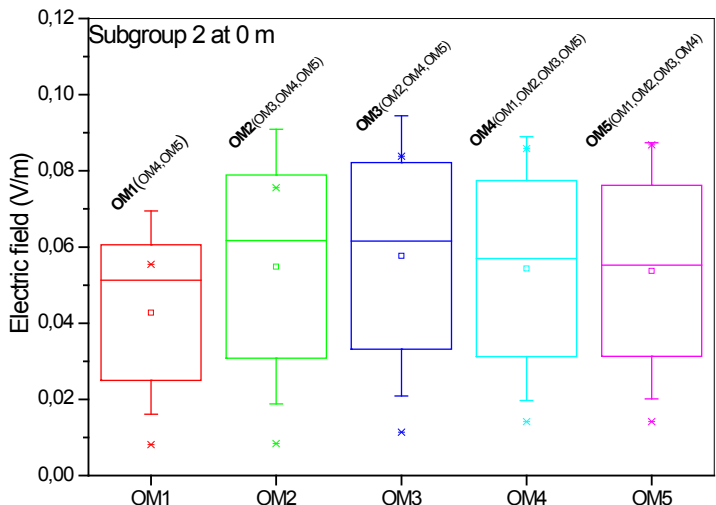

Figure 2: Box-Whisker plots of EMR intensity at $0 \mathrm{~m}$ for the nine (9) mobile devices of Subgroup 2 at the five different operation modes (OM1 to OM5).

Overall, the above reported results showed that for the mobile devices of Subgroup 1 the mean EMR intensity at $0 \mathrm{~m}$ exhibited a strong dependence on the operation mode employed.

Subgroup 2: It included nine (9) mobile devices. The mean EMR values of the devices in this subgroup were in the range between 0.008 $\mathrm{V} / \mathrm{m}$ and $0.087 \mathrm{~V} / \mathrm{m}$ under all OMs. The individual spectra in at least one of the five OMs showed a few isolated peaks with intensities ranging between $0.1 \mathrm{~V} / \mathrm{m}$ and $1 \mathrm{~V} / \mathrm{m}$. In general, the mean EMR values of this subgroup showed a modest change as a function of the OM employed. More specifically, by dividing the maximum with the minimum mean EMR value among the five mean EMR values obtained under each OM sub-data for each specific mobile device, a factor ranging between 1.17 and 1.75 was obtained (maximal change as a function of $\mathrm{OM}$ )

Table 4 presents the main descriptive statistics for the data of Subgroup 2 at a distance of $0 \mathrm{~m}$ from the device.

All values are well below the limit values of Table 2. Similarly to the case of Subgroup 1, RM-ANOVA was used in order to test for statistically significant differences between the total means as a function of the operation mode for the devices belonging to Subgroup 2 .

The RM-ANOVA showed a statistically significant effect of the operation mode $(\mathrm{OM})$ on the EMR intensity emitted by the mobile devices belonging to Subgroup 2 at distance equal to $0 \mathrm{~m}(F(2.131$, $17.050)=8.486, p=0.002)$. The pairwise comparison via post-hoc tests (Bonferroni correction) showed the following specific statistically significant differences: OM1 $<\mathrm{OM} 2 \quad(p=0.015)$ and $\mathrm{OM} 1<\mathrm{OM} 3$ $(p=0.010)$. In other words, there is a small (by a factor equal to ca. 1.3) statistically significant increase in mean EMR intensity at $0 \mathrm{~m}$ in operation modes 2 (ringing/call effort) and 3 (usage) relative to operation mode 1 (long term inactivity). The mean EMR intensities in operation modes 4 and 5 are shown to be statistically similar to all the other OMs.

In Figure 2, the mean EMR values obtained for the 9 mobile devices of Subgroup 2 are depicted in the form of Box-Whisker plots. The mode of presentation is similar to the one employed in Figure 1. Overall, the above reported results showed that for the mobile devices of Subgroup 2 the mean EMR intensity at 0 m exhibited a weak dependence on the operation mode employed.

Subgroup 3: It included nineteen (19) mobile devices. The mean EMR values of the devices in this subgroup were in the range between $0.007 \mathrm{~V} / \mathrm{m}$ and $0.009 \mathrm{~V} / \mathrm{m}$ under all OMs. In this subgroup, the individual spectra in at least one of the five OMs showed isolated 
Citation: Koulougliotis D, Nikolopoulos D, Gorgolis N, Karidas L, Petraki E, et al. (2018) Effect of the Operation Mode and Distance on the Electromagnetic Radiation Emitted by Mobile Phone Devices in Greece: A Pilot Study. J Civil Environ Eng 8: 300. doi: 10.4172/2165$784 X .1000300$

small peaks with intensities always lower than $0.1 \mathrm{~V} / \mathrm{m}$. In general, the mean EMR values of this subgroup remained low and showed small (however systematic) changes as a function of the OM employed. Thus, we obtained maximal changes (in all OMs) by factors which ranged between 1.04 and 1.14 .

The corresponding descriptive statistics are shown in Table 5. All these values are well below the limit values of Table 2 .

In a similar manner as for Subgroups 1 and 2, RM-ANOVA was used in order to test for statistically significant differences between the total means as a function of the operation mode for the devices belonging to Subgroup 3.

The RM-ANOVA showed the existence of a statistically significant effect of the operation mode on the EMR intensity emitted by the mobile devices belonging to Subgroup 3 at distance equal to $0 \mathrm{~m}$ $(F(1.930,34.736)=97.787, p<0.001)$. The Pairwise comparisons via post-hoc tests (Bonferroni correction) showed the following specific statistically significant differences:

OM1<OM2 $(p<0.001)$, OM3 $(p<0.001)$, OM4 $(p<0.001)$, OM5 $(p<0.001)$

$$
\begin{aligned}
& \text { OM2 }<\text { OM3 }(p=0.001), \text { OM4 }(p<0.001), \text { OM5 }(p<0.001) \\
& \text { OM3 }<\text { OM4 }(p=0.009), \text { OM5 }(p<0.001) \\
& \text { OM4<OM5 }(p<0.001)
\end{aligned}
$$

More specifically, there is a statistically significant increase in EMR intensity by ca. a factor of 1.05 on passing from the OM1 to OM2 and a further increase by ca. a factor of 1.01 on passing from OM2 to OM3, from OM3 to OM4 and from OM4 to OM5. These results of the pairwise comparisons may be summarised as follows:

\section{$\mathrm{OM} 1<\mathrm{OM} 2<\mathrm{OM} 3<\mathrm{OM} 4<\mathrm{OM} 5$}

Overall, the mobile devices in Subgroup 3 showed low levels of EMR intensity with a gradual increase as a function of operation mode which was statistically significant but very small (the largest factor is ca. equal to 1.09 and takes place between OM5 and OM1).

In Figure 3, the mean EMR values obtained for the 19 mobile devices of Subgroup 3 are depicted in the form of Box-Whisker plots together with the results of the statistical analysis via RM-ANOVA. The mode of presentation was similar to the one employed in Figures 1 and 2.

\section{EMR intensity of mobile devices at $1 \mathbf{~ m}$}

Subsequently, the EMR intensity of the mobile devices in the

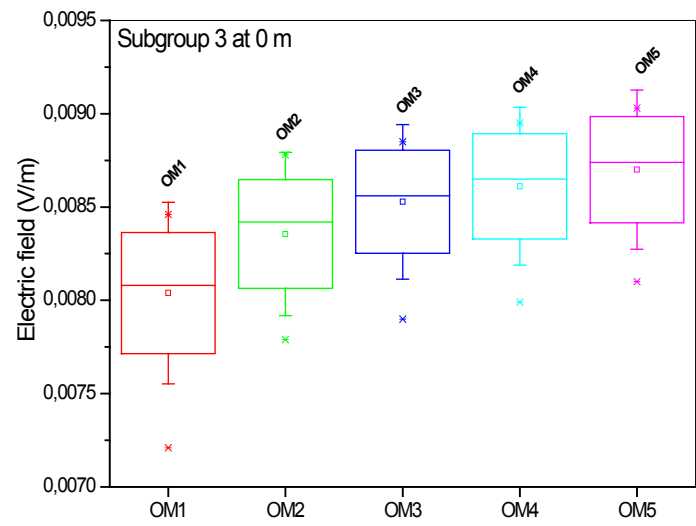

Figure 3: Box-Whisker plots of EMR intensity at $0 \mathrm{~m}$ for the nineteen (19) mobile devices of Subgroup 3 at the five different operation modes (OM1 to OM5).

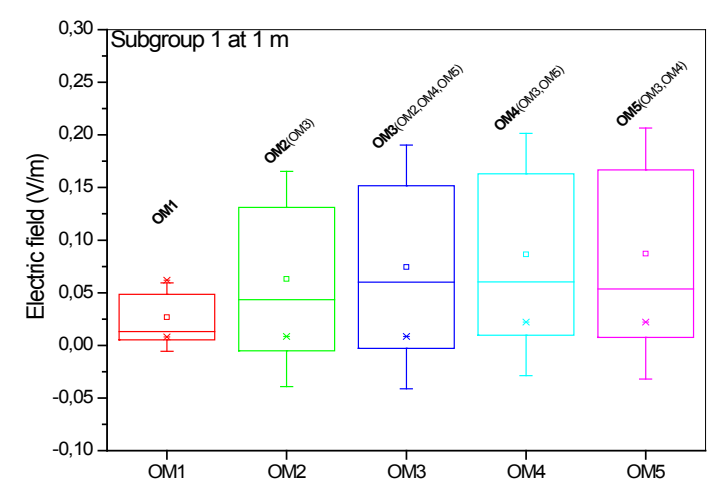

Figure 4: Box-Whisker plots of EMR intensity at $1 \mathrm{~m}$ for the twenty-four (24) mobile devices of Subgroup 1 at the five different operation modes (OM1 to OM5).

different operation modes at a distance equal to $1 \mathrm{~m}$ from the device was investigated. The results are reported separately for the same Subgroups 1,2 and 3 that were identified above.

Subgroup 1: The four data sets OM2, OM3, OM4 and OM5 were shown to satisfy the Kolomogorov-Smirnov criterion for normality ( $p>0.05$ ) while the one corresponding to OM1 was shown not to meet this condition $(p=0.028)$. The corresponding descriptive statistics are shown in Table 6.

All values of Table 6 are well below the limit values of Table 2 .

The application of RM-ANOVA showed a statistically significant effect of the operation mode on the EMR intensity emitted by the mobile devices belonging to Subgroup 1 at distance equal to $1 \mathrm{~m}$ $(F(1.526,35.108)=13.797, p<0.001)$. The pairwise comparisons via post-hoc tests (Bonferroni correction) showed the following specific statistically significant differences:

OM1 $<$ OM2 $(p=0.049)$, OM3 $(p=0.020)$, OM4 $(p=0.002)$ and OM5 $(p=0.004)$

$\mathrm{OM} 2<\mathrm{OM} 4(p=0.006)$ and OM5 $(p=0.039)$

More specifically, a statistically significant increase in EMR intensity was detected by ca. a factor of 2.3 on passing from the OM1 to OM2. On passing from OM2 to OM3 an increase by ca. a factor of 1.17 was not statistically significant $(p=0.291)$. Absence of statistical significance was also found upon passing from OM3 to OM4 ( $p=0.102)$ as well as from OM3 to OM5 ( $p=0.411)$ with increases by factors of ca. 1.16 and 1.18, respectively, However, a statistically significant increase was detected by ca. a factor of 1.37 upon passing from OM2 to OM4 $(p=0.0046)$ and of ca. 1.38 from OM2 to OM5 ( $p=0.039)$.

In Figure 4, the mean EMR values obtained for the 24 mobile devices of Subgroup 1 are depicted in the form of Box-Whisker plots together with the results of the statistical analysis via RM-ANOVA. The mode of presentation is similar to the one employed in Figure 1.

Subgroup 2: All five data sets (one for each $\mathrm{OM}$ ) were shown to satisfy the Kolmogorov-Smirnov criterion for normality. The total means and corresponding standard deviations for each OM are shown in Table 7.

All the values are well below the limit values of Table 2 .

The use of RM-ANOVA showed no statistically significant effect of the operation mode $(\mathrm{OM})$ on the EMR intensity emitted by the mobile devices belonging to Subgroup 2 at distance equal to $1 \mathrm{~m}(F(1.414$, $11.316)=3.693, p=0.070$ ).

In Figure 5, the mean EMR values obtained for the 9 mobile devices 
Citation: Koulougliotis D, Nikolopoulos D, Gorgolis N, Karidas L, Petraki E, et al. (2018) Effect of the Operation Mode and Distance on the Electromagnetic Radiation Emitted by Mobile Phone Devices in Greece: A Pilot Study. J Civil Environ Eng 8: 300. doi: 10.4172/2165784X.1000300

of Subgroup 2 are depicted in the form of Box-Whisker plots together with the results of the statistical analysis via RM-ANOVA. The mode of presentation is the same as the one employed in Figure 1.

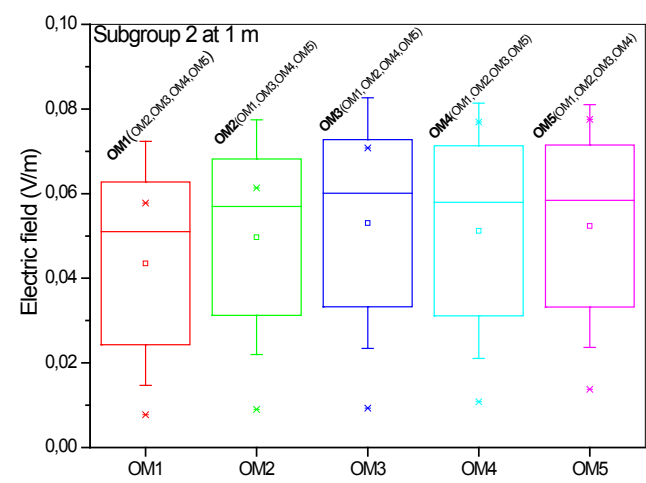

Figure 5: Box-Whisker plots of EMR intensity at $1 \mathrm{~m}$ for the nine (9) mobile devices of Subgroup 2 at the five different operation modes (OM1 to OM5).

\begin{tabular}{|c|c|c|}
\hline Operation Mode & Total Mean (V/m) & Std. Dev. (V/m) \\
\hline OM1 & 0.008 & 0.0003 \\
\hline OM2 & 0.0084 & 0.0003 \\
\hline OM3 & 0.0085 & 0.0003 \\
\hline OM4 & 0.0086 & 0.0003 \\
\hline OM5 & 0.0087 & 0.0003 \\
\hline
\end{tabular}

Table 5: Descriptive Statistics for Subgroup $3(\mathrm{~N}=19$, Distance $=0 \mathrm{~m})$.

\begin{tabular}{|c|c|c|}
\hline Operation Mode & Total Mean $\mathbf{( V / m )}$ & Std. Dev. $\mathbf{( V / m})$ \\
\hline OM1 & 0.027 & 0.022 \\
\hline OM2 & 0.063 & 0.068 \\
\hline OM3 & 0.074 & 0.077 \\
\hline OM4 & 0.086 & 0.077 \\
\hline OM5 & 0.087 & 0.08 \\
\hline
\end{tabular}

Table 6: Descriptive Statistics for Subgroup $1(\mathrm{~N}=24$, Distance=1 m).

\begin{tabular}{|c|c|c|}
\hline Operation Mode & Total Mean $(\mathbf{V} / \mathbf{m})$ & Std. Dev. $\mathbf{( V / m})$ \\
\hline OM1 & 0.044 & 0.019 \\
\hline OM2 & 0.05 & 0.018 \\
\hline OM3 & 0.053 & 0.02 \\
\hline OM4 & 0.051 & 0.02 \\
\hline OM5 & 0.052 & 0.019 \\
\hline
\end{tabular}

Table 7: Descriptive Statistics for Subgroup $2(\mathrm{~N}=9$, Distance=1 $\mathrm{m})$.

\begin{tabular}{|c|c|c|}
\hline Operation Mode & Total Mean $\mathbf{( V / m )}$ & Std. Dev. (V/m) \\
\hline OM1 & 0.008 & 0.0003 \\
\hline OM2 & 0.0083 & 0.0003 \\
\hline OM3 & 0.0084 & 0.0003 \\
\hline OM4 & 0.0085 & 0.0003 \\
\hline OM5 & 0.0086 & 0.0003 \\
\hline
\end{tabular}

Table 8: Descriptive statistics for subgroup $3(\mathrm{~N}=19$, Distance $=1 \mathrm{~m})$.

\begin{tabular}{|c|c|c|}
\hline Ratio of Total Means & Distance $=\mathbf{0} \mathbf{~ m}$ & Distance=1 $\mathbf{~ m}$ \\
\hline OM2/OM1 & 5.3 & 2.37 \\
\hline OM3/OM1 & 7.13 & 2.74 \\
\hline OM4/OM1 & 8.8 & 3.18 \\
\hline OM5/OM1 & 8.8 & 3.22 \\
\hline OM3/OM2 & 1.35 & 1.17 (n.s.) \\
\hline OM4/OM2 & 1.66 & 1.37 \\
\hline OM5/OM2 & 1.66 & 1.38 \\
\hline
\end{tabular}

Table 9: Ratios of total means between EMR intensities of operation modes with pairwise statistically significant differences in either distance (Subgroup 1).

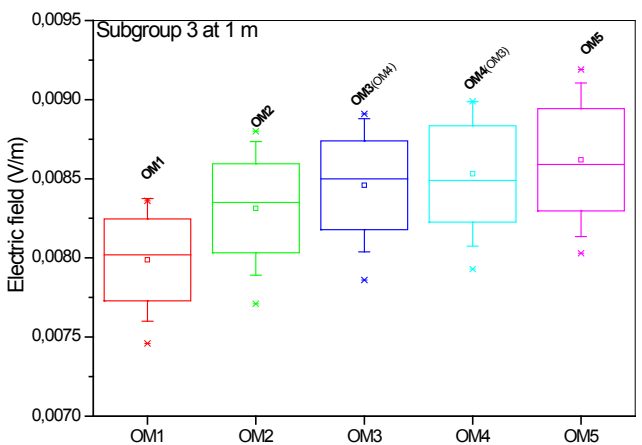

Figure 6: Box-Whisker plots of EMR intensity at $1 \mathrm{~m}$ for the nineteen (19) mobile devices of Subgroup 3 at the five different operation modes (OM1 to OM5).

Subgroup 3: All five data sets (one for each OM) were shown to satisfy the Kolmogorov-Smirnov criterion for normality. The total means and corresponding standard deviations for each OM are shown in Table 8 .

All the values are well below the limit values of Table 2 .

The application of RM-ANOVA showed that there exists a statistically significant effect of the operation mode on the EMR intensity emitted by the mobile devices belonging to Subgroup 3 at distance equal to $1 \mathrm{~m}(F(1.549,27.882)=142.655, p<0.001)$. The pairwise comparisons via post-hoc tests (Bonferroni correction) showed the following specific statistically significant differences:

OM1<OM2 $(p<0.001)$, OM3 $(p<0.001)$, OM4 $(p<0.001)$, OM5 $(p<0.001)$

OM2 $<$ OM3 $(p=0.001)$, OM4 $(p<0.001)$, OM5 $(p<0.001)$

OM3 $<$ OM5 $(p=0.001)$

OM4 $<$ OM5 $(p<0.001)$

More specifically, there was a statistically significant increase in EMR intensity by ca. a factor of 1.05 on passing from the OM1 to OM2 and a further increase by ca. a factor of 1.02 on passing from OM2 to OM3. There was no statistical difference upon passing from OM3 to OM4 $(p=0.105)$. Furthermore, there was a statistically significant increase by ca. a factor of 1.01 on passing from OM4 to OM5. These results of the pairwise comparisons may be summarised as follows:

\section{$\mathrm{OM} 1<\mathrm{OM} 2<\mathrm{OM} 3 \approx \mathrm{OM} 4<\mathrm{OM} 5$}

Overall, the mobile devices in Subgroup 3 showed low levels of EMR intensity with a gradual increase as a function of operation mode which was statistically significant but very small (the largest factor was ca. equal to 1.08 between OM5 and OM1).

In Figure 6, the mean EMR values obtained for the 19 mobile devices of Subgroup 3 are depicted in the form of Box-Whisker plots together with the results of the statistical analysis via RM-ANOVA. The mode of presentation is similar to the one employed in Figure 1.

\section{Comparison of EMR intensity between two distances $(0 \mathrm{~m}$ vs $1 \mathrm{~m})$}

In this section, a comparison of the EMR intensity for the mobile devices of each subgroup measured at $0 \mathrm{~m}$ and $1 \mathrm{~m}$ is reported.

Subgroup 1: The ratios of the total mean maximal EMR intensities between the operation modes which showed pairwise statistically 
Citation: Koulougliotis D, Nikolopoulos D, Gorgolis N, Karidas L, Petraki E, et al. (2018) Effect of the Operation Mode and Distance on the Electromagnetic Radiation Emitted by Mobile Phone Devices in Greece: A Pilot Study. J Civil Environ Eng 8: 300. doi: 10.4172/2165$784 X .1000300$

Page 6 of 9

significant differences at either of the two examined distances are shown in Table 9. It is observed that these ratios were lowered (by percentages ranging between $13 \%$ and $64 \%$ ) as the distance increased from $0 \mathrm{~m}$ to $1 \mathrm{~m}$. This provides evidence that at longer distances the dependence of the EMR intensity on the operation mode tends to be attenuated. At the longer distance, all statistically significant differences between the operation modes are maintained with the exception of the one between OM3 and OM2.

In Table 10, the ratios of the total means of the EMR intensities at the two distances $(0 \mathrm{~m}$ and $1 \mathrm{~m})$ are given together with the results of the paired samples t-test between the two distances for each operation. As shown in Table 10, as the distance increased, the total means of the EMR intensity of the mobile devices belonging to Subgroup 1 decreased significantly (by factors ranging between 2.5 and 3.0) in all operation modes employed, with the exception of OM1. Similar results were

\begin{tabular}{|c|c|}
\hline Operation Mode & Ratio of Total Means: $\mathbf{0 ~} \mathbf{~} / \mathbf{1} \mathbf{~ m}$ \\
\hline OM1 & $1.11($ n.s. $)(p=0.400)$ \\
\hline OM2 & $2.52(p=0.001)$ \\
\hline OM3 & $2.89(p<0.001)$ \\
\hline OM4 & $3.07(p<0.001)$ \\
\hline OM5 & $3.03(p<0.001)$ \\
\hline
\end{tabular}

Table 10: Results of the paired samples t-test between the two distances for each operation mode for the mobile devices of Subgroup 1.

\begin{tabular}{|c|c|c|}
\hline Ratio of Total Means & Distance=0 $\mathbf{~ m}$ & Distance=1 $\mathbf{~ m}$ \\
\hline OM2/OM1 & 1.28 & 1.14 (n.s.) \\
\hline OM3/OM1 & 1.35 & 1.20 (n.s.) \\
\hline
\end{tabular}

Table 11: Ratios of total means between EMR intensities of operation modes with pairwise statistically significant differences in either distance (Subgroup 2).

\begin{tabular}{|c|c|}
\hline Operation Mode & Ratio of Total Means: $\mathbf{0} \mathbf{~ m} / \mathbf{1 ~} \mathbf{~}$ \\
\hline OM1 & 0.98 (n.s.) $(p=0.475)$ \\
\hline OM2 & 1.10 (n.s. $)(p=0.122)$ \\
\hline OM3 & 1.09 (n.s.) $(p=0.252)$ \\
\hline OM4 & 1.06 (n.s.) $(p=0.445)$ \\
\hline OM5 & 1.04 (n.s.) $(p=0.647)$ \\
\hline
\end{tabular}

Table 12: Results of the paired samples t-test between the two distances for each operation mode for the mobile devices of Subgroup 2.

\begin{tabular}{|c|c|c|}
\hline Ratio of Total Means & Distance= $\mathbf{~ m}$ & Distance=1 $\mathbf{~}$ \\
\hline OM2/OM1 & 1.04 & 1.04 \\
\hline OM3/OM1 & 1.06 & 1.06 \\
\hline OM4/OM1 & 1.07 & 1.07 \\
\hline OM5/OM1 & 1.08 & 1.08 \\
\hline OM3/OM2 & 1.02 & 1.02 \\
\hline OM4/OM2 & 1.03 & 1.03 \\
\hline OM5/OM2 & 1.04 & 1.04 \\
\hline OM4/OM3 & 1.009 & 1.008 (n.s.) \\
\hline OM5/OM3 & 1.02 & 1.02 \\
\hline OM5/OM4 & 1.01 & 1.01 \\
\hline
\end{tabular}

Table 13: Ratios of total means between EMR intensities of operation modes with pairwise statistically significant differences in either distance (Subgroup 3).

\begin{tabular}{|c|c|}
\hline Operation Mode & Ratio of Total Means: $\mathbf{0 m} / \mathbf{1 m}$ \\
\hline OM1 & 1.006 (n.s.) $(p=0.349)$ \\
\hline OM2 & 1.005 (n.s.) $(p=0.317)$ \\
\hline OM3 & 1.008 (n.s. $)(p=0.151)$ \\
\hline OM4 & 1.009 (n.s. $)(p=0.130)$ \\
\hline OM5 & 1.009 (n.s.) $(p=0.116)$ \\
\hline
\end{tabular}

Table 14: Results of the paired samples t-test between the two distances for each operation mode for the mobile devices of Subgroup 3. reached upon application of the non-parametric Wilcoxon signedranks test as well.

Subgroup 2: The ratios of the total mean maximal EMR intensities between the operation modes which exhibited pairwise statistically significant differences at either of the two examined distances are shown in Table 11. As also observed in Subgroup 1, these ratios tend to be smaller as the distance increases thus providing evidence for an attenuation of the dependence of the EMR intensity on the operation mode at longer distances. For the mobile devices of this subgroup this effect was also evidenced from the fact that at $1 \mathrm{~m}$ there were no statistically significant changes of the EMR intensity as a function of the operation mode.

In Table 12 the ratios of the total means of the EMR intensities at the two distances $(0 \mathrm{~m}$ and $1 \mathrm{~m})$ are given together with the results of the paired samples t-test between the two distances for each operation mode. The fact that the ratios have values near 1 in combination with the large $p$-values $(>0.05)$ of the statistical test, provide evidence that in all operation modes, the total means of the EMR intensities observed for the mobile devices of Subgroup 2 at the two measured distances are statistically similar. Note that non-statistically significant differences between the two distances were found upon application of the nonparametric Wilcoxon signed-ranks test as well.

Subgroup 3: The ratios of the total mean maximal EMR intensities between the operation modes which showed pairwise statistically significant differences at either of the two examined distances, are shown in Table 13. All ratios, with the exception of OM4/OM3 did not show any significant change as the distance increased. All statistically significant differences between the operation modes were found for both distances with the exception of the one between OM3 and OM4 at $1 \mathrm{~m}$.

In Table 14 the ratios of the EMR intensities at the two distances $(0 \mathrm{~m}$ and $1 \mathrm{~m})$ are given together with the results of the paired samples t-test between the two distances for each operation mode are given. The results are similar to the ones obtained for the mobile devices of Subgroup 2, i.e. in all operation modes, the EMR intensities observed for the mobile devices of Subgroup 3 at the two measured distances do no differ significantly.

\section{Discussion}

This pilot study aimed at investigating the dependence of the EMR intensity emitted by mobile phone devices on two factors, namely a) the operation mode of the device and $b$ ) the distance from the device. Measurements were conducted in five different operation modes and two distances in a total of 52 mobile devices.

The first important finding of the current study has been the identification of three distinct categories/subgroups of mobile devices displaying unique and also sharing some common characteristics. The discussion will first concentrate on the results reached from the measurements at distance equal to $0 \mathrm{~m}$.

At $0 \mathrm{~m}$ all three subgroups showed a statistically significant effect of the operation mode on the emitted EMR intensity. This effect was stronger in Subgroup 1 (maximal changes by factors ranging between 2.47 and 45.8), followed by Subgroup 2 (maximal increases by factors in the range $1.17-1.75$ ) and by Subgroup 3 (maximal increases by factors in the range $1.04-1.14$ ). As far as the absolute level of the emitted EMR intensity is concerned, the observed lowest individual mean EMR values were similar in all three subgroups $(0.007 \mathrm{~V} / \mathrm{m}$ for Subgroups 1 and 3, $0.008 \mathrm{~V} / \mathrm{m}$ for Subgroup 2) and they were displayed at the 
Citation: Koulougliotis D, Nikolopoulos D, Gorgolis N, Karidas L, Petraki E, et al. (2018) Effect of the Operation Mode and Distance on the Electromagnetic Radiation Emitted by Mobile Phone Devices in Greece: A Pilot Study. J Civil Environ Eng 8: 300. doi: 10.4172/2165$784 X .1000300$

Page 7 of 9

same operating mode, namely OM1 (long term inactivity). However, the observed highest individual mean EMR values were dramatically different between subgroups with the following order:

Subgroup $1(=0.82 \mathrm{~V} / \mathrm{m})>$ Subgroup $2(=0.087 \mathrm{~V} / \mathrm{m})>$ Subgroup 3 $(=0.009 \mathrm{~V} / \mathrm{m})$

A similar trend was shown also for the intensities of the peaks appearing in the individual spectra of the devices of each subgroup. More specifically, all devices of Subgroup 1 displayed large peaks $(>1$ $\mathrm{V} / \mathrm{m}$ ) in the spectra of at least one of the five operations modes. On the other hand, the peak intensity was lower for the devices of Subgroup 2 (between $0.1 \mathrm{~V} / \mathrm{m}$ and $1 \mathrm{~V} / \mathrm{m}$ ) and even weaker forro those of Subgroup 3 (always $<0.1 \mathrm{~V} / \mathrm{m}$ ).

It should be pointed out, that in all subgroups and under all operation modes the measured electric field strength values (either those of the peaks in the individual spectra, the individual mean values for each devices or the total means) remained well below the exposure limits of $41 \mathrm{~V} / \mathrm{m}-61 \mathrm{~V} / \mathrm{m}$ which are recommended by ICNIRP for the general public $[24,25]$. However, if one takes into account the more stringent limits (of ca. $4 \mathrm{~V} / \mathrm{m}$ ) established by several EU member states in order to address mostly requirements for more sensitive population groups [24], it should be mentioned that the individual spectra of the majority of the devices of Subgroup 1 (19 out of 24 devices) displayed peaks larger than $4 \mathrm{~V} / \mathrm{m}$ (and up to $26 \mathrm{~V} / \mathrm{m}$ ) in all operation modes at $0 \mathrm{~m}$ except for OM1. More specifically, such peaks were observed for $10,12,18$ and 19 devices in operation modes OM2, OM3, OM4 and OM5 respectively.

A closer examination on the qualitative characteristics of the effect of the operation mode on the EMR intensity emitted by mobile devices at $0 \mathrm{~m}$ shows the following:

i) The transition from OM1 (long term inactivity) to OM2 (Ringing/Call effort) is the only one-step transition which results to a systematic increase in the intensity of EMR emitted by the mobile devices. The increase is larger in Subgroup 1, quite less in Subgroup 2 and even less in Subgroup 3. The mean increase factors are 5.30, 1.28 and 1.04 for Subgroups 1, 2 and 3 respectively. In addition, these mean increase factors are the highest observed for all one-step transitions in all subgroups

ii) The subsequent one-step transition from OM2 (Ringing/Call effort) to OM3 (phone usage-during the conversation) results to an increase in the emitted EMR intensity for mobile devices belonging to Subgroups 1 and 3 (by mean increase factors of 1.35 and 1.02 respectively), while no statistically significant difference was observed for the devices of Subgroup 2.

iii) In further transitions (OM3 to OM4 and OM5) i.e. right or shortly after the end of the call, the emitted EMR intensity either remains in an increased level which is similar (Subgroup 1) or slightly higher (Subgroup 3) than what is observed in OM3, or it returns to the level observed in OM1 (Subgroup 2).

iv) Besides OM1 $\rightarrow$ OM2, another transition which also results to a systematic increase in the emitted EMR intensity, is the two-step transition from OM1 (long term inactivity) to OM3 (phone usageduring the conversation), with a similar trend between subgroups. More specifically, the mean increase factors are 7.13, 1.35 and 1.06 for Subgroups 1, 2 and 3 respectively.

v) All one-step transitions of Subgroup 3 show statistically significant increases in the emitted EMR intensity which are however very small in magnitude. Thus the total mean increase observed between OM1 and OM5 is less than 10\%. The devices belonging to this subgroup display low levels of emitted EMR intensity, irrespective of the operation mode employed, which range between $0.007 \mathrm{~V} / \mathrm{m}$ and $0.009 \mathrm{~V} / \mathrm{m}$.

Moving on to the results reached from the measurements at distance equal to $1 \mathrm{~m}$, the following remarks can be made: At $1 \mathrm{~m}$ only two subgroups (namely 1 and 3 ) showed a statistically significant effect (increase) of the operation mode on the emitted EMR intensity, with the effect being much stronger in Subgroup 1. More specifically, by examining the change in the total means the largest ones observed were by factors equal to 3.22 and 1.08 for the transition OM1 $\rightarrow$ OM5 in Subgroups 1 and 3 respectively. At $0 \mathrm{~m}$, these factors equal to 8.80 and 1.08 for Subgroups 1 and 3 respectively. It is thus deduced that at distance equal to $1 \mathrm{~m}$, the effect of the operation mode on the emitted EMR intensity continued to exist, however attenuated relative to the one at $0 \mathrm{~m}$.

Examination of the distance dependence of the emitted EMR intensity showed no statistically significant differences between the two distances employed $(0 \mathrm{~m}$ and $1 \mathrm{~m})$ for the mobile devices belonging to Subgroups 2 and 3 under all operation modes. On the other hand, a statistically significant decrease (by a factor between 2 and 3 ) in emitted EMR intensity with increasing distance $(0 \mathrm{~m} \rightarrow 1 \mathrm{~m})$ was observed for the mobile devices of Subgroup 1 for all operation modes except for OM1 (long term inactivity).

\section{Conclusions}

The results of the present work may be summarized with the following conclusions:

a) The electromagnetic radiation (EMR) emitted by mobile phone devices is dependent on the type of the device. Based on the observed peak intensity in the individual experimental spectra (electric field vs frequency) three specific device subgroups were identified in the current study (Intensities of Subgroup 1>Subgroup 2>Subgroup 3).

b) EMR emitted by mobile phone devices is dependent on the operation mode of the device. The onset of usage either by an incoming or an outgoing call ("ringing/call effort") from "long term inactivity" induces a systematic increase in the level of emitted EMR which can be either very small (ca. $4 \%$ for Subgroup 3) or quite significant (by a factor $>5$ for Subgroup 1). A similar systematic increase (by factors ranging between 1.06 and $>7$ ) in the emitted EMR relative to the initial state of "long term inactivity" is observed upon continuation of device usage.

c) EMR emitted by mobile phone devices is dependent on the distance from the device for the device subgroup characterized by the peaks of highest intensity (Subgroup 1). Upon increasing the distance from $0 \mathrm{~m}$ to $1 \mathrm{~m}$ a significant decrease is observed in the emitted EMR (by a factor between 2 and 3) for all operation modes except for the initial one ("long term inactivity").

Finally, it should be pointed out that even though the present study was conducted in a limited number of mobile devices, it gave significant insights to the dependence of the intensity of the RF electromagnetic radiation emitted by mobile phone devices on the operating conditions and the distance. More extensive measurements are required in the future in order to validate the current research findings and examine more detailed aspects of the research questions posed.

\section{References}

1. Ahlbom A, Bridges J, deSeze R, Hillert L, Juutilainen J, et al. (2008) Possible effects of electromagnetic fields (EMF) on human health-opinion of the 
Citation: Koulougliotis D, Nikolopoulos D, Gorgolis N, Karidas L, Petraki E, et al. (2018) Effect of the Operation Mode and Distance on the Electromagnetic Radiation Emitted by Mobile Phone Devices in Greece: A Pilot Study. J Civil Environ Eng 8: 300. doi: 10.4172/2165784X.1000300

Page 8 of 9

scientific committee on emerging and newly identified health risks (SCENIHR). Toxicology 246:248-250.

2. HPA (Health Protection Agency) (2004) Advice on limiting exposure to electromagnetic fields $(0-300 \mathrm{GHz})$. Report of the independent Advisory Group on non-ionizing radiation.

3. HPA (Health Protection Agency) (2012). Health effects from radiofrequency electromagnetic fields. Report of the independent Advisory Group on nonionizing radiation.

4. ICNIRP (International Commission on Non-lonizing Radiation Protection) (2002) General approach to protection against non-ionizing radiation. Health Phys 82:540-548.

5. AGNIRP Health effects from radiofrequency electromagnetic fields (2003) Report of an independent Advisory. Group on Non-ionising Radiation. Doc NRPB 14: 1-77.

6. SCENIHR (Scientific Committee on Emerging and Newly Identified Health Risks) (2007) Possible effects of Electromagnetic Fields (EMF) on Human Health. Brussels: European Commission.

7. SCENIHR (Scientific Committee on Emerging and Newly Identified Health Risks) (2009). Health Effects of Exposure to EMF. Brussels, European Commission

8. SCENIHR (Scientific Committee on Emerging and Newly Identified Health Risks) (2013) Preliminary opinion on Potential health effects of exposure to electromagnetic fields (EMF). Brussels, European Commission.

9. Valberg PA, van Deventer TE, Repacholi MH (2007) Workgroup report: base stations and wireless networks-radiofrequency (RF) exposures and health consequences. Environ Health Perspect 115:416-424.

10. IEEE (Institute of Electrical and Electronics Engineers) (2004) Standard for local and metropolitan area networks Part 16: air interface for fixed broadband wireless access systems. 2004. Piscataway, NY, IEEE, IEEE 802,16d.

11. IEEE (Institute of Electrical and Electronics Engineers) (2005) Standard for local and metropolitan area networks part 16: air interface for fixed and mobile broadband wireless access systems. Amendment 2: physical and medium access control layers for combined fixed and mobile operation in licensed bands and corrigendum 1 corrigendum to IEEE Std 802, 16-2004 (Revision of IEEE Std 802.16-2001). Piscataway, NY, IEEE 802,16e.

12. IEEE (Institute of Electrical and Electronics Engineers) (2005) Standard for safety levels with respect to human exposure to radio frequency electromagnetic fields, $3 \mathrm{kHz}$ to $300 \mathrm{GHz}$. Piscataway, NY, IEEE, IEEE C95.1

13. IEC (International Electrotechnical Commission) (2004) Exposure to electric or magnetic fields in the low and intermediate frequency range-Methods for calculating the current density and internal electric field induced in the human body. Geneva, IEC 62226.

14. IEC (International Electrotechnical Commission) (2005) Measurement methods for electromagnetic fields of household appliances and similar apparatus with regard to human exposure. Geneva, IEC 62233.

15. IEC (International Electrotechnical Commission) (2005) Medical electrica equipment-part 1: general requirements for safety; 2: collateral standard: electromagnetic compatibility-requirements and tests. Geneva, IEC 60601-1-2.

16. IEC (International Electrotechnical Commission) (2005) Human Exposure to Radio Frequency Fields from Hand-Held and Body-Mounted Wireless Communication Devices - Human Models, Instrumentation, and Procedures to Determine the Specific Absorption Rate (SAR) for Hand-Held Devices Used in Close Proximity to the Ear (Frequency Range of $300 \mathrm{MHz}$ to $3 \mathrm{GHz}$ ). Geneva, IEC 62209.

17. WHO (World Health Organization) (2002) Establishing a dialogue on risks from electromagnetic fields. Geneva, Switzerland.

18. WHO (World Health Organization) (2006) Electromagnetic fields and public health: base stations and wireless technologies. Geneva, Switzerland.

19. WHO (World Health Organization) (2006) WHO Research Agenda for Radiofrequency Fields. Geneva, Switzerland.

20. WHO (World Health Organization) (2010) WHO Research Agenda for Radiofrequency Fields. Geneva, Switzerland.

21. WHO (World Health Organization) (2011) Electromagnetic fields and public health: mobile phones. Fact sheet $N^{\circ} 193$. Geneva, Switzerland.
22. CEU (Council of the European Union) (1999) Council Recommendation of 12 July 1999 on the limitation of exposure of the general public to electromagnetic fields $(0 \mathrm{~Hz}$ to $300 \mathrm{GHz})$. L199/59.

23. European Parliament and CEU (Council of the European Union) (2013) Directive 2013/35/EU.

24. Stam R (2011) Comparison of international policies on electromagnetic fields (power frequency and radiofrequency fields). Laboratory for Radiation Research, National Institute for Public Health and the Environment, the Netherlands. RIVM 118/2011 LSO Sta.

25. Stuchly MA (1979) Interaction of radiofrequency and microwave radiation with living systems. A review of mechanisms. Radiat Environ Biophys 16: 1-14

26. ICNIRP (International Commission on Non-lonizing Radiation Protection) (2010) Guidelines for limiting exposure to time-varying electric and magnetic fields (1 Hz to $100 \mathrm{kHz}$ ). Health Phys 99:818-836.

27. ICNIRP (International Commission on Non-lonizing Radiation Protection) (2009) Statement on the "Guidelines for limiting exposure to time-varying electric, magnetic and electromagnetic fields (up to $300 \mathrm{GHz}$ ). Health Phys 97:257-258.

28. Ahlbom A, Bergqvist U, Bernhardt JH, Cesarini JP, Grandolfo M, et al. (1998) Guidelines for limiting exposure to time-varying electric, magnetic, and electromagnetic fields (up to $300 \mathrm{GHz}$ ). Health Phys 74: 494-521.

29. ICNIRP (International Commission on Non-lonizing Radiation Protection) (2003) Guidance on determining compliance of exposure to pulsed and complex non-sinusoidal waveforms below $100 \mathrm{kHz}$ with ICNIRP guidelines. Health Phys 84:383-387.

30. ICNIRP (International Commission on Non-lonizing Radiation Protection) (2003) Exposure to static and low frequency electromagnetic fields, biological effects and health consequences $(0-100 \mathrm{kHz})$. In: Bernhardt $\mathrm{JH}$, Matthes $\mathrm{R}$, McKinlay A, Vecchia P, Veyret B (eds). Review of the scientific evidence and health consequences. 2003 Munich: ICNIRP.

31. Van Leeuwen GM, Lagendijk JJ, Van Leersum BJ, Zwamborn AP, Hornsleth $\mathrm{SN}$, et al. (1999) Calculation of change in brain temperatures due to exposure to a mobile phone. Phys Med Biol 44: 2367-2379.

32. Viel JF, Clerc S, Barrera C, Rymzhanova R, Moissonnier M, et al. (2009) Residential exposure to radiofrequency fields from mobile phone base stations, and broadcast transmitters: a population-based survey with personal meter. Occup Environ Med 66: 550-556.

33. Vrijheid M, Armstrong BK, Bedard D, Brown J, Deltour I, et al. (2008) Recall bias in the assessment of exposure to mobile phones. J Expo Sci Environ Epidemiol 19: 369-381.

34. Wainwright $P(2000)$ Thermal effects of radiation from cellular telephones. Phys Med Biol 45: 2363-2372.

35. Thomas S, Kühnlein A, Heinrich S, Praml G, Nowak D, et al (2008) Persona exposure to mobile phone frequencies and well-being in adults: A crosssectional study based on dosimetry. Bioelectromagnetics 29: 463-470.

36. Thuróczy G, Molnar F, Janosy G, Nagy N, Kubinyi G, et al. (2008) Personal RF exposimetry in urban area. Ann Telecommun 63: 87-96.

37. Timmel CR, Till U, Brocklehurst B, McLauchlan KA, Hore PJ (1998) Effects of weak magnetic fields on free radical recombination reactions. Mol Phys 95: 71-89.

38. Sheppard AR, Swicord ML, Balzano Q (2008) Quantitative evaluations of mechanisms of radiofrequency interactions with biological molecules and processes. Health Phys 95: 365-396.

39. Lauer O, Frei P, Gosselin MC, Joseph W, Röösli M, et al. (2013) Combining Near and Far Field exposure for an organ-specific and whole-body RF-EMF proxy for epidemiological research: a reference case. Bioelectromagnetics 34 : 366-374.

40. Joseph W, Verloock L, Goeminne F, Vermeeren G, Martens L (2012) Assessment of RF exposures from emerging wireless communication technologies in different environments. Health Phys 102: 161-172.

41. Joseph W, Verloock $L$ (2010) Influence of mobile phone traffic on base station exposure of the general public. Health Phys 99: 631-638

42. Hardell L, Näsman A, Påhlson A, Hallquist A, Mild KH (1999) Use of cellular telephones and the risk for brain tumours: A case- control study. Int $\mathrm{J}$ Oncol 15: 113-116. 
Citation: Koulougliotis D, Nikolopoulos D, Gorgolis N, Karidas L, Petraki E, et al. (2018) Effect of the Operation Mode and Distance on the Electromagnetic Radiation Emitted by Mobile Phone Devices in Greece: A Pilot Study. J Civil Environ Eng 8: 300. doi: 10.4172/2165784X.1000300

43. Hardell L, Nasman A, Pahlson A, Hallquist A (2000) Case-control study on radiology work, medical $\mathrm{x}$-ray investigations, and use of cellular telephones as risk factors for brain tumors. Med Gen Med.

44. Hardell L, Mild KH, Påhlson A, Hallquist A (2001) lonizing radiation, cellular telephones and the risk for brain tumours. Eur J Cancer Prev 10: 523-529.

45. Hardell L, Hallquist A, Mild KH, Lagendijk JJ, Van Leersum BJ, et al. (2002) Cellular and cordless telephones and the risk for brain tumours. Eur $\mathrm{J}$ Cancer Prev 11: 377-386.

46. Hardell L, Mild KH, Carlberg M (2002) Case-control study on the use of cellular and cordless phones and the risk for malignant brain tumours. Int J Radiat Bio 78: 931-936.

47. Hardell L, Mild KH, Carlberg M (2003) Further aspects on cellular and cordless telephones and brain tumours. Int J Oncol 22: 399-407.

48. Hardell L, Carlberg M, Mild KH (2006) Pooled analysis of two case-control studies on the use of cellular and cordless telephones and the risk of benign brain tumours diagnosed during 1997-2003. Int J Oncol 28: 509-518.

49. Hardell L, Carlberg M, Mild KH (2006) Pooled analysis of two case-control studies on use of cellular and cordless telephones and the risk for malignant brain tumours diagnosed in 1997-2003. Int Arch Occup Environ Health 79: 630639 .

50. Hardell L, Carlberg M (2009) Mobile phones, cordless phones and the risk for brain tumours. Int J Oncol 35: 5-17.
51. Hardell L, Carlberg M, Mild KH (2009) Epidemiological evidence for an association between use of wireless phones and tumor diseases. Pathophysiology 16: 113-122

52. Hardell L, Carlberg M, Mild KH (2010) Mobile phone use and the risk for malignant brain tumors: a case-control study on deceased cases and controls. Neuroepidemiology 35: 109-114.

53. Hardell L, Carlberg M, Mild KH (2011) Pooled analysis of case-control studies on malignant brain tumours and the use of mobile and cordless phones including living and deceased subjects. Int J Oncol 38: 1465-1474.

54. Georgiou CD (2010) Oxidative stress-induced biological damage by low-leve EMFs: mechanism of free radical pair electron-spin polarization and biochemical amplification. In: Non-thermal effects and mechanisms of interaction between electromagnetic fields and living matter. ICEMS Monograph, Giuliani L, Soffritti M, editors. Bologna, Italy: National Institute for the Study and Control of Cancer and Environmental Diseases "Bernardino Ramazzini". pp. 63-113.

55. Gupta MK, Khanna RK, Rangra KJ, Vijay YK (2018) Interaction of cell phone radiations and human nervous system. Adv Intelligent Sys Comput 632: 413-419.

56. Jadia S, Qureshi S, Jain L, Shringirishi M (2018) Adverse effect of mobile phone on hearing in healthy individuals: A clinical study. Indian J Otolaryngo Head Neck Surg 1-5.

57. Baby NM, Koshy G, Mathew A (2017) The effect of electromagnetic radiation due to mobile phone use on thyroid function in medical students studying in a Medical College in South India. Indian J Endocrinol Metab 21:797-802. 